\title{
RELACIÓN ENTRE VARIABLES METEOROLÓGICAS E INCENDIOS FORESTALES EN LA PROVINCIA PINAR DEL RÍO, CUBA
}

\author{
Marcos Pedro Ramos Rodríguez ${ }^{1}$, Yulian Carrasco Rodríguez ${ }^{2}$, Carlos Alberto Miranda Sierra ${ }^{3}$, \\ Antonio Carlos Batista ${ }^{4}$, Alexandre França Tetto ${ }^{4}$ \\ ${ }^{1}$ UNESUM, Jipijapa, Manabí, Ecuador - marcos.ramos@unesum.edu.ec \\ ${ }^{2}$ UPR, Departamento Forestal, Pinar del Río, Cuba - yulian@upr.edu.cu \\ ${ }^{3}$ Centro Meteorológico, Pinar del Río, Cuba - calberto@ pri.insmet.cu \\ ${ }^{4}$ UFPR, Departamento de Ciencias Forestales, Curitiba, Paraná, Brasil - batistaufpr@ufpr.br; tetto@ufpr.br
}

Recibido para publicación: 24/02/2017 - Aceito para publicación: 18/09/2017

\begin{abstract}
Resumen
Comprender la relación existente entre variables meteorológicas e incendios forestales es importante con vistas a una gestión de las actividades propias del manejo integral del fuego dentro de límites económicamente viables, socialmente aceptables y ecológicamente apropiados. Esta investigación fue desarrollada con el objetivo de argumentar las relaciones existentes entre variables meteorológicas tales como temperatura del aire, humedad relativa, precipitación y velocidad del viento y los incendios forestales en la provincia Pinar del Río, Cuba. La base de datos meteorológicos utilizados fue facilitada por el Centro Meteorológico Provincial de Pinar del Río y los datos de incendios forestales fueron obtenidos en el Cuerpo de Guardabosques de Pinar del Río a través de los correspondientes registros de ocurrencias de incendios. Toda la información utilizada se refiere al período del 01/01/2010 al 31/12/2014, totalizando cinco años de observaciones. Para los análisis estadísticos se utilizó el programa informático IBM SPSS Statistic versión 22. Los resultados muestran que la distribución anual de las variables meteorológicas tiene relación directa o inversa con la distribución de las ocurrencias de incendios y las áreas quemadas en la provincia Pinar del Río durante el período 2010-2014. Independientemente de lo anterior, las correlaciones entre variables meteorológicas e incendios forestales fueron bajas o muy bajas. No obstante, es posible demostrar que el mes de abril es el más propicio para la ocurrencia de los incendios forestales.

Palabras clave: Manejo del fuego; prevención de incendios forestales; factores meteorológicos; propagación del fuego; combustible forestal disponible.
\end{abstract}

\begin{abstract}
Relationship between meteorological variables and forest fires in the province Pinar del Rio, Cuba. Understanding the relationship between forest fires and meteorological variables is important with a view to managing the activities of the integrated fire management within economically viable, socially acceptable and environmentally sound limits. This research was developed with the aim of argument the relationships between some meteorological variables and forest fires in the province Pinar del Rio, Cuba. The basis of meteorological data used was provided by the Provincial Meteorological Center of Pinar del Río through the corresponding fire occurrence records. The variables considered were air temperature, relative humidity, rainfall and wind speed. Forest fire data were obtained in the Forest Guard Corps of Pinar del Rio. All information used refers to the period from $01 / 01 / 2010$ to $31 / 12 / 2014$, totaling five years of observations. Computer program SPSS Statistic version 22 was utilized for the statistics analysis. The results show that the annual distribution of meteorological variables have direct or inverse relation to the distribution of fire occurrences and burned areas in the province Pinar del Río during the period 2010-2014. Notwithstanding the above, the correlations between meteorological variables and forest fires were low or very low. However, it is possible to show that the month of April is the most favorable for the occurrence of forest fires.

Keywords: Fire management; forest fire prevention; meteorological factors; fire spread; forest fuel available.
\end{abstract}

\section{INTRODUCCIÓN}

La mayor parte de los incendios forestales tienen un origen antrópico, sin embargo, no todos los días ocurren incendios ni siempre estos muestran el mismo comportamiento. Esto sucede porque tanto la ocurrencia como la propagación, dependen en gran medida de las condiciones meteorológicas, las cuales además de variar durante el día y el año, pueden hacerlo también, en cortos periodos de tiempo. Debido a esto los índices de peligro de incendios más utilizados en el mundo, utilizan alguna variable meteorológica.

FLORESTA, Curitiba, PR, v. 47, n. 3, p. 343 - 352, jul. / set. 2017

Rodríguez, M. P. R. et al.

ISSN eletrônico 1982-4688

DOI: $10.5380 /$ rf.v47i1.50900 
Varios investigadores tienen verificada la estrecha relación entre los incendios forestales y los factores meteorológicos e indican que todos los grandes incendios forestales han ocurrido durante condiciones climáticas extremas y anormales. Sequías prolongadas acompañadas por altas temperaturas del aire y bajas humedades relativas, determinan las condiciones para los más violentos incendios, por la reducción del contenido de humedad de los combustibles forestales a niveles anormalmente bajos (MOLINA-TERRÉN; CARDIL, 2015; THOMPSON; SPIES, 2009; LIU et al., 2010).

La posibilidad y la frecuencia de ocurrencia de los incendios forestales para Soares y Batista (2007) están íntimamente relacionadas con las condiciones meteorológicas. Estos autores agregan que por ese motivo, el conocimiento de algunas variables meteorológicas es de gran importancia en la prevención de los incendios.

La ocurrencia de los incendios forestales en una región es afectada por las condiciones de las capas más bajas de la atmósfera en un determinado tiempo y por las alteraciones climáticas ocurridas con el paso del tiempo. Esos cambios ocurren debido a la naturaleza de la atmósfera y de la reacción a la recepción de la energía emitida por el sol. Por este motivo, el conocimiento de las variables meteorológicas es de gran importancia en la prevención de los incendios. A través de las variables meteorológicas se pueden identificar los días y épocas de mayor probabilidad de ocurrencia de incendios y así se pueden tomar medidas técnicas y administrativas para reducir el potencial de daños por el fuego (SOARES; BATISTA, 2007; SOARES et al., 2015).

De acuerdo con Westerling y Bryant (2008) el clima afecta el riesgo de incendios forestales principalmente a través de sus efectos sobre la disponibilidad de humedad. Las condiciones de humedad durante la estación de crecimiento de los combustibles, especialmente combustible fino (hierbas, etc.) estimulan la producción a través del crecimiento de la vegetación, mientras que las condiciones secas durante y antes de la temporada de incendios aumentan la inflamabilidad de la vegetación viva y muerta que alimenta los incendios forestales. La disponibilidad de humedad es una función de la precipitación acumulada y de la temperatura.

La importancia de los factores meteorológicos en la actividad de incendios forestales es bien conocida y la habilidad para anticipar su impacto sobre la ocurrencia diaria de incendios y el comportamiento ha sido un objetivo importante para los investigadores desde el inicio de la ciencia del fuego. Como resultado de ello, un gran número de métodos de evaluación de peligro de incendios, generada exclusivamente sobre la base de propiedades meteorológicas se han desarrollado (VIEGAS et al., 1999).

En correspondencia con todo lo expuesto anteriormente, puede decirse que las variables meteorológicas influyen en la distribución diaria y anual de las ocurrencias de los incendios forestales y las áreas quemadas, ya sea de forma directa sobre el fuego o indirectamente cambiando el estado de los combustibles forestales. Resulta entonces importante y necesario entender la magnitud y características de esa influencia para la toma adecuada de decisiones en el ámbito de las actividades propias del manejo integral del fuego. En correspondencia con todo lo anterior, esta investigación se desarrolló con el objetivo de argumentar las relaciones existentes entre variables meteorológicas tales como temperatura del aire, humedad relativa, precipitación y velocidad del viento y los incendios forestales en la provincia Pinar del Río, Cuba, probándose la hipótesis de que en el periodo del estudio, las variables meteorológicas tienen relación con la ocurrencia y propagación de los incendios forestales.

\section{MATERIALES Y MÉTODOS}

\section{Caracterización del área de estudio}

El trabajo se desarrolló en la provincia Pinar del Río, Cuba, en la cual se reporta el mayor porcentaje de ocurrencias de incendios forestales en el país. Esta provincia tiene una superficie de $8883,74 \mathrm{~km}^{2}$ y la misma está situada entre $\operatorname{los} 21^{\circ} 45^{\prime}$ y $22^{\circ} 55^{\prime}$ de latitud norte y entre los $83^{\circ} 04^{\prime}$ y $84^{\circ} 57^{\prime}$ de longitud oeste (Figura 1). Según el Servicio Estatal Forestal (2012) citado por Carrasco (2016) el 45,7 \% del área de la provincia está cubierta por bosques, siendo la de mayor superficie boscosa del país. En la misma los bosques naturales, las plantaciones establecidas y las jóvenes cubren superficies de 286 836,90; 106 916,50 y 16 177,30 ha, respectivamente. Estas plantaciones son en su gran mayoría de Pinus spp., Eucalyptus spp. y Casuarina spp., ocurriendo precisamente en ellas la mayor cantidad de los incendios forestales de la provincia.

Durante el periodo 2010-2014 los valores medios anuales de la precipitación, la temperatura del aire, la humedad relativa y la velocidad del viento fueron de $1482,40 \mathrm{~mm}, 24,8{ }^{\circ} \mathrm{C}, 77 \%$ y $6 \mathrm{~km} . \mathrm{h}^{-1}$, respectivamente. Según la clasificación climática de Köppen, el clima en la provincia es Aw (clima tropical con mes más frío con temperatura mayor de $18{ }^{\circ} \mathrm{C}$ y una estación seca de al menos un mes con menos de $60 \mathrm{~mm}$ ). La administración y manejo del recurso forestal en la provincia son desarrollados por siete Empresas Forestales Integrales (EFI) organizadas sobre el territorio de uno o de varios municipios, nivel político-administrativo en que se divide la provincia. La estructura del sistema de manejo del fuego incluye un Departamento Provincial, siete circuitos de protección de montaña y un territorio de protección. Los circuitos y el territorio se organizan en Brigadas Profesionales de Prevención y Combate a los Incendios Forestales y Grupos de Trabajo divididos estos últimos en Áreas de Responsabilidad. 


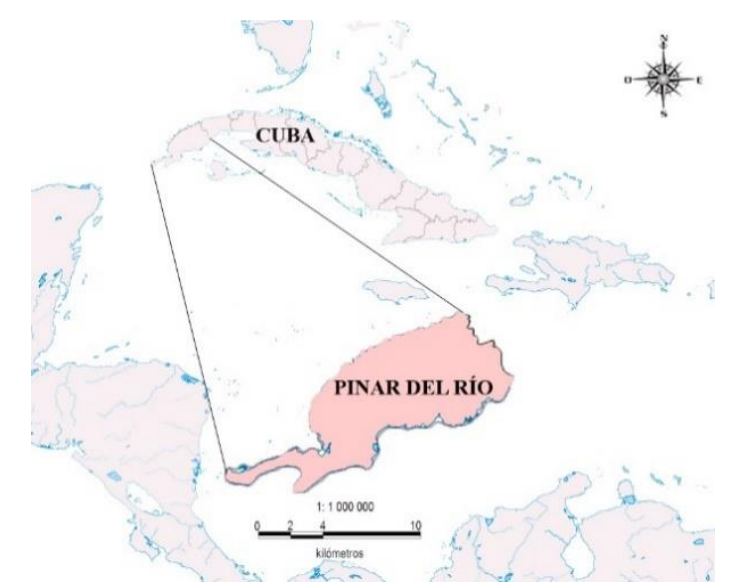

Figura 1. Ubicación de la provincia Pinar del Río.

Figure 1. Location of the Pinar del Río province.

\section{Análisis experimentales}

Como parte de los análisis experimentales se consideró tanto la distribución de las variables meteorológicas como de la ocurrencia y propagación de los incendios forestales, además de las relaciones entre unas y otras variables. Todos los datos se refieren al período del 01/01/2010 al 31/12/2014, totalizando cinco años de observaciones.

La distribución de las variables meteorológicas se analizó considerando la distribución de sus valores medios mensuales. En todos los casos se hizo una descripción estadística y se compararon los valores diarios medios mensuales de cada variable determinándose la correlación existente entre las mismas. La base de datos meteorológicos utilizados fue facilitada por el Centro Meteorológico Provincial de Pinar del Río. Las variables consideradas fueron temperatura del aire, humedad relativa, precipitación y velocidad del viento. En el caso de la precipitación, las mediciones correspondieron a los acumulados diarios y para el resto de las variables se consideraron las mediciones diarias registradas a las 13:00 horas.

El análisis de la distribución del número de ocurrencias de incendios y las respectivas áreas quemadas se realizó considerando los valores medios mensuales de estas variables. Los datos de incendios forestales fueron obtenidos en el Cuerpo de Guardabosques de Pinar del Río a través de los correspondientes registros de ocurrencias de incendios los cuales son llenados para cada incendio, conteniendo información sobre fechas, horas, tipo de incendio, área quemada, fuerzas y medios que participaron en las labores de extinción y pérdidas económicas. También se determinó las correlaciones de las variables meteorológicas con el número de ocurrencias y las áreas quemadas.

\section{Tratamiento estadístico}

La comparación de medias se realizó utilizando un total de 60 observaciones, correspondientes estas a las medias mensuales de cada uno de los cinco años del período utilizado para la investigación. Para verificar la normalidad de los datos correspondientes a cada una de las variables, se utilizó la prueba de normalidad de Kolmogorov-Smirnov. Solo los datos correspondientes a la humedad relativa se ajustaron a la distribución normal $(\mathrm{p}=0,200)$. En correspondencia con esto, para comparar los valores medios mensuales, se utilizó un análisis de varianza (ANOVA) para la humedad relativa y para el resto de las variables, cuyos datos no se ajustaron a la distribución normal ( $\mathrm{p}<0,05)$, la prueba no paramétrica $\mathrm{H}$ de Kruskal-Wallis. En ambos casos se utilizaron para cada mes cinco valores medios, correspondiente cada uno a los cinco años del periodo considerado para la investigación. A posteriori se llevó a cabo la identificación de los meses cuyos valores medios fueron diferentes en el periodo analizado. Para esto se utilizaron las pruebas de comparaciones múltiples de Tukey para la humedad relativa y de Dunn para el resto de las variables.

Para determinar la relación entre las variables se utilizaron las 1826 observaciones correspondientes a cada uno de los días del periodo objeto de análisis. De acuerdo con la prueba de normalidad de KolmogorovSmirnov, ninguna variable analizada siguió una distribución normal con $p=0,000$. En consecuencia con esto la relación entre las mismas se determinó a través del coeficiente de correlación no paramétrica de Spearman, considerando una probabilidad de significación del 5\%. La interpretación de los valores de la correlación fue hecha de acuerdo con la propuesta de Bisquerra (2004), la cual se muestra en la tabla 1.

FLORESTA, Curitiba, PR, v. 47, n. 3, p. 343 - 352, jul. / set. 2017 
Tabla 1. Interpretación de los valores de las correlaciones de Spearman.

Table 1. Interpretation of values Spearman correlations.

\begin{tabular}{cc}
\hline Valor de $\mathbf{r}$ & Interpretación \\
\hline 0,00 & Nula \\
$0,01-0,19$ & Muy baja \\
$0,20-0,39$ & Baja \\
$0,40-0,59$ & Moderada \\
$0,60-0,79$ & Alta \\
$0,80-0,99$ & Muy alta \\
1,00 & Perfecta \\
\hline
\end{tabular}

El procesamiento de la base de datos sobre incendios forestales se realizó con el Sistema Integrado para el Manejo de Bases de Datos sobre Incendios Forestales versión 1.2 (SIMBDIF v.1.2.) desarrollado por Rodríguez (RODRÍGUEZ et al., 2014). Los análisis estadísticos se hicieron con el programa estadístico informático IBM SPSS Statistic versión 22.

\section{RESULTADOS}

En la tabla 2 se muestra el análisis descriptivo de los valores diarios de las variables meteorológicas en Pinar del Río durante los cinco años del estudio (2010-2014). Puede observarse que los mayores valores del coeficiente de variación se obtuvieron para la precipitación, lo cual se puede explicar por su desigual distribución diaria a través de los meses. No todos los días llueve y tampoco es siempre la misma cantidad.

Tabla 2. Análisis descriptivo de los valores diarios de las variables meteorológicas a través de los meses en Pinar del Río (2010-2014).

Table 2. Monthly statistics of the meteorological variables in Pinar del Río province (2010-2014).

\begin{tabular}{|c|c|c|c|c|c|c|c|c|c|c|c|c|c|c|c|c|}
\hline \multirow[t]{2}{*}{ Meses } & \multicolumn{4}{|c|}{$\begin{array}{c}\text { Temperatura } \\
\left({ }^{\circ} \mathrm{C}\right)\end{array}$} & \multicolumn{4}{|c|}{$\begin{array}{c}\text { Humedad relativa } \\
(\%)\end{array}$} & \multicolumn{4}{|c|}{$\begin{array}{c}\text { Precipitación } \\
(\mathrm{mm})\end{array}$} & \multicolumn{4}{|c|}{$\begin{array}{c}\text { Velocidad viento } \\
\left(\mathrm{m}^{\left.-\mathrm{s}^{-1}\right)}\right.\end{array}$} \\
\hline & Min & Max & $\bar{X}$ & $\mathrm{CV}$ & Min & Max & $\bar{X}$ & $\mathrm{CV}$ & Min & Max & $\bar{X}$ & $\mathrm{CV}$ & Min & Max & $\bar{X}$ & $\mathrm{CV}$ \\
\hline Ene & 12,1 & 25,5 & 21,78 & 0,12 & 52,7 & 97,5 & 75,05 & 0,12 & 0,0 & 17,0 & 1,10 & 2,85 & 0,3 & 17,3 & 4,00 & 0,88 \\
\hline Feb & 15,7 & 26,5 & 23,32 & 0,10 & 54,0 & 93,3 & 72,31 & 0,12 & 0,0 & 15,9 & 1,22 & 2,47 & 0,0 & 20,8 & 4,15 & 0,87 \\
\hline Mar & 17,6 & 27,6 & 23,70 & 0,10 & 50,2 & 96,7 & 66,53 & 0,14 & 0,0 & 37,1 & 1,64 & 3,22 & 0,3 & 21,2 & 5,89 & 0,65 \\
\hline Abr & 19,1 & 28,7 & 26,07 & 0,06 & 46,8 & 93,2 & 68,94 & 0,13 & 0,0 & 40,4 & 2,02 & 2,67 & 0,7 & 18,7 & 5,44 & 0,58 \\
\hline May & 23,2 & 30,2 & 26,98 & 0,05 & 46,7 & 94,2 & 72,27 & 0,14 & 0,0 & 22,9 & 3,47 & 1,57 & 0,7 & 16,0 & 4,70 & 0,66 \\
\hline Jun & 22,3 & 30,8 & 27,50 & 0,07 & 59,7 & 95,6 & 78,45 & 0,11 & 0,0 & 147,0 & 8,16 & 2,32 & 0,3 & 14,7 & 3,83 & 0,73 \\
\hline Jul & 22,5 & 30,9 & 27,58 & 0,05 & 63,0 & 91,2 & 77,57 & 0,08 & 0,0 & 57,0 & 4,80 & 1,58 & 0,3 & 19,0 & 3,16 & 0,83 \\
\hline Ago & 24,0 & 30,4 & 27,57 & 0,06 & 65,5 & 94,2 & 80,25 & 0,09 & 0,0 & 40,8 & 5,69 & 1,32 & 0,0 & 17,5 & 2,71 & 0,88 \\
\hline Sep & 23,4 & 29,5 & 26,67 & 0,05 & 67,8 & 93,8 & 83,54 & 0,06 & 0,0 & 47,9 & 4,46 & 1,48 & 0,0 & 10,0 & 2,00 & 0,82 \\
\hline Oct & 20,7 & 27,4 & 25,34 & 0,05 & 61,8 & 97,8 & 82,11 & 0,10 & 0,0 & 64,8 & 4,70 & 2,17 & 0,0 & 15,5 & 3,30 & 0,94 \\
\hline Nov & 17,1 & 26,5 & 23,16 & 0,08 & 59,7 & 94,2 & 78,09 & 0,10 & 0,0 & 24,9 & 1,40 & 2,80 & 0,0 & 14,2 & 3,40 & 0,80 \\
\hline Dic & 14,9 & 26,0 & 22,13 & 0,11 & 52,0 & 93,2 & 77,40 & 0,11 & 0,0 & 20,2 & 0,97 & 2,97 & 0,3 & 14,7 & 3,34 & 0,87 \\
\hline
\end{tabular}

Min: Mínimo; Max: Máximo; $\bar{X}$ : media; CV: Coeficiente de variación

Los menores valores diarios promedios de la precipitación a través de los meses se presentaron durante el periodo noviembre-abril. Resulta interesante destacar que durante los últimos cuatro meses de este periodo, se observaron también los mayores valores diarios promedios de la velocidad del viento, mientras que durante febrero y abril, últimos meses del periodo mencionado, las medias mensuales de la humedad relativa son también menores. Estas tres condiciones unidas determinan la disponibilidad de los combustibles forestales.

Durante el mes de abril, último del periodo poco lluvioso, se presentan altos valores de temperatura del aire y de velocidades del viento, mientras que los correspondientes a la humedad relativa y la precipitación son bajos.

El análisis de varianza aplicado a los valores medios de la humedad relativa demostró que entre los mismos existe diferencia significativa $(\mathrm{p}=0,000)$. La prueba no paramétrica de Kruskal-Wallis, aplicada al resto de las variables, permitió establecer que en todos los casos los valores medios mensuales para el período analizado son también diferentes $(\mathrm{p}=0,000)$. Los resultados de las pruebas estadísticas de Tukey en el caso de la humedad relativa y Dunn, para el resto de las variables, se muestran en la tabla 3. 
Tabla 3. Resultados de las pruebas de Tukey y Dunn para comparación mensual de medias.

Table 3. Results of Tukey and Dunn tests for monthly comparison of means.

\begin{tabular}{ccccc}
\hline Meses & Temperatura del aire $\left({ }^{\circ} \mathbf{C}\right)$ & Humedad relativa $(\%)$ & Precipitación $(\mathbf{m m})$ & Velocidad del viento $\left(\mathbf{m} . \mathbf{s}^{\mathbf{- 1}}\right)$ \\
\hline Ene & $21,78^{\mathrm{a}}$ & $75,05^{\mathrm{d}}$ & $1,10^{\mathrm{b}}$ & $4,00^{\mathrm{de}}$ \\
Feb & $23,32^{\mathrm{c}}$ & $72,31^{\mathrm{c}}$ & $1,22^{\mathrm{b}}$ & $4,15^{\mathrm{e}}$ \\
Mar & $23,70^{\mathrm{c}}$ & $66,53^{\mathrm{a}}$ & $1,64^{\mathrm{c}}$ & $5,89^{\mathrm{h}}$ \\
Abr & $26,07^{\mathrm{e}}$ & $68,94^{\mathrm{b}}$ & $2,02^{\mathrm{c}}$ & $5,44^{\mathrm{g}}$ \\
May & $26,98^{\mathrm{g}}$ & $72,27^{\mathrm{c}}$ & $3,47^{\mathrm{d}}$ & $4,70^{\mathrm{f}}$ \\
Jun & $27,50^{\mathrm{h}}$ & $78,45^{\mathrm{f}}$ & $8,16^{\mathrm{g}}$ & $3,83^{\mathrm{d}}$ \\
Jul & $27,58^{\mathrm{h}}$ & $77,57^{\mathrm{e}}$ & $4,80^{\mathrm{ef}}$ & $3,16^{\mathrm{c}}$ \\
Ago & $27,57^{\mathrm{h}}$ & $80,25^{\mathrm{g}}$ & $5,69^{\mathrm{f}}$ & $2,71^{\mathrm{b}}$ \\
Sep & $26,67^{\mathrm{f}}$ & $83,54^{\mathrm{h}}$ & $4,46^{\mathrm{e}}$ & $2,00^{\mathrm{a}}$ \\
Oct & $25,34^{\mathrm{d}}$ & $82,11^{\mathrm{g}}$ & $4,70^{\mathrm{e}}$ & $3,30^{\mathrm{c}}$ \\
Nov & $23,16^{\mathrm{c}}$ & $78,09^{\mathrm{f}}$ & $1,40^{\mathrm{b}}$ & $3,40^{\mathrm{c}}$ \\
Dic & $22,13^{\mathrm{b}}$ & $77,40^{\mathrm{e}}$ & $0,97^{\mathrm{a}}$ & $3,34^{\mathrm{c}}$ \\
\hline
\end{tabular}

Medias seguidas de las mismas letras en las columnas no difieren entre sí por el test de Tukey para la humedad relativa y por el test de Dunn para el resto de las variables $(p \leq 0,05)$

Durante el período 2010-2014 en la provincia Pinar del Río ocurrieron 378 incendios y las áreas quemadas ascendieron a 7837,11 ha de bosques, mostrando medias anuales de 75,6 incendios y 1567,42 ha, respectivamente. Durante dicho período, entre los meses de marzo a junio, ocurrió el 65,18\% de los incendios concentrándose el 90,06\% de las áreas quemadas entre marzo y mayo. Estos periodos incluyen los últimos meses del periodo poco lluvioso, en los cuales la mayor cantidad del material combustible se encuentra disponible, y primeros meses del periodo lluvioso, en los cuales todavía existe material combustible disponible y donde además, se origina gran cantidad de incendios por rayos que acompañan las precipitaciones. La distribución de las medias mensuales de ocurrencias de incendios y áreas quemadas en Pinar del Río (2010-2014) se muestran en la figura 2.

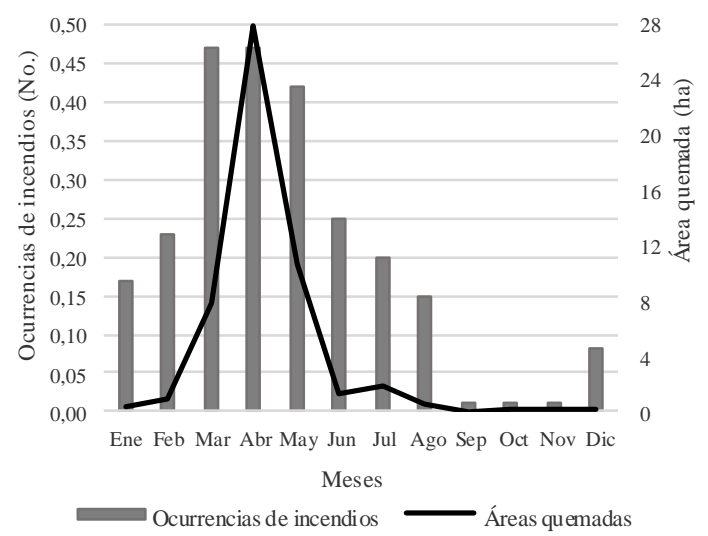

Figura 2. Distribución de las medias mensuales de ocurrencias de incendios y áreas quemadas en Pinar del Río (2010-2014).

Figure 2. Distribution of monthly mean of occurrences of fires and burned areas in Pinar del Río (2010-2014).

La prueba estadística no paramétrica $\mathrm{H}$ de Kruskal-Wallis demostró la existencia de diferencia significativa entre las medias obtenidas para cada uno de los meses con valores de $\mathrm{p}=0,000$ y 0,001 para las ocurrencias de incendios y las áreas quemadas, respectivamente. En la tabla 4 se observa que los altos valores medios registrados para las ocurrencias de incendios en abril son similares solo a los obtenidos en marzo, mientras que en el caso de las áreas quemadas, el valor es diferente al resto de los meses. Todo esto puede ser consecuencia del comportamiento de las variables meteorológicas en el área de estudio, según se ha tratado en párrafos anteriores. Cabe mencionar que para ambas variables, se han utilizado valores medios diarios para cada uno de los meses durante los cinco años analizados.

FLORESTA, Curitiba, PR, v. 47, n. 3, p. 343 - 352, jul. / set. 2017

Rodríguez, M. P. R. et al.

ISSN eletrônico 1982-4688

DOI: $10.5380 /$ rf.v47i1.50900 
Tabla 4. Distribución mensual de ocurrencias de incendios y áreas quemadas en Pinar del Río (2010-2014) y resultados de la prueba de Dunn para comparación mensual de medias.

Table 4. Monthly distribution of forest fire occurrence and burned areas in Pinar del Río (2010-2014) and results of Dunn tests for monthly comparison of means.

\begin{tabular}{ccccccrrr}
\hline \multirow{2}{*}{ Meses } & \multicolumn{3}{c}{ Ocurrencias de incendios (No.) } & \multicolumn{4}{c}{ Áreas quemadas (ha) } \\
\cline { 2 - 9 } & Min & Max & $\bar{X}$ & CV & Min & \multicolumn{1}{c}{ Max } & \multicolumn{1}{c}{} & CV \\
\hline Ene & 0 & 2 & $0,17^{\mathrm{c}}$ & 2,40 & 0 & 15,00 & $0,32^{\mathrm{d}}$ & 4,54 \\
$\mathrm{Feb}$ & 0 & 5 & $0,23^{\mathrm{c}}$ & 2,83 & 0 & 59,40 & $0,99^{\mathrm{d}}$ & 5,94 \\
Mar & 0 & 5 & $0,47^{\mathrm{f}}$ & 1,88 & 0 & 629,70 & $7,90^{\mathrm{f}}$ & 7,11 \\
Abr & 0 & 6 & $0,47^{\mathrm{f}}$ & 1,74 & 0 & 3627,00 & $28,00^{\mathrm{g}}$ & 10,59 \\
May & 0 & 5 & $0,42^{\mathrm{e}}$ & 2,17 & 0 & 262,40 & $10,60^{\mathrm{f}}$ & 3,91 \\
Jun & 0 & 5 & $0,25^{\mathrm{d}}$ & 2,75 & 0 & 40,00 & $1,22^{\mathrm{e}}$ & 4,36 \\
Jul & 0 & 6 & $0,20^{\mathrm{c}}$ & 3,58 & 0 & 131,87 & $1,80^{\mathrm{e}}$ & 6,30 \\
Ago & 0 & 3 & $0,15^{\mathrm{c}}$ & 3,11 & 0 & 14,00 & $0,47^{\mathrm{d}}$ & 4,05 \\
Sep & 0 & 1 & $0,01^{\mathrm{a}}$ & 8,00 & 0 & 1,00 & $0,01^{\mathrm{a}}$ & 12,36 \\
Oct & 0 & 1 & $0,01^{\mathrm{a}}$ & 8,20 & 0 & 15,20 & $0,10^{\mathrm{b}}$ & 12,25 \\
Nov & 0 & 1 & $0,01^{\mathrm{a}}$ & 8,20 & 0 & 15,20 & $0,10^{\mathrm{b}}$ & 12,25 \\
Dic & 0 & 3 & $0,08^{\mathrm{a}}$ & 4,16 & 0 & 7,00 & $0,12^{\mathrm{c}}$ & 5,60 \\
\hline Medias seguidas de las mismas letras en las columnas no difieren entre sí por el test de Dunn $(p \leq 0,05)$ & &
\end{tabular}

En la figura 3 puede observarse que durante los meses en los cuales los valores medios de la temperatura son mayores no es cuando son mayores los valores medios de las ocurrencias de incendios. Esto evidencia que no es la temperatura la variable que determina el comportamiento de los incendios, al menos directamente o por sí sola. La humedad relativa muestra los valores más bajos de marzo a mayo, meses donde también se registran los valores medios más altos de la velocidad del viento. En el caso de la precipitación, los menores valores promedio diarios se presentan durante los meses de noviembre a abril. En este caso los cuatro primeros meses de sequía llevan al estrés hídrico de la vegetación herbácea con la consecuente muerte de una gran parte de la misma. De esta forma en marzo y abril las condiciones son ideales para la ocurrencia y propagación del fuego, situación que se mantiene en mayo a pesar de que el valor medio registrado para la precipitación es diferente estadísticamente con los valores de los seis meses anteriores. Esto se explica porque aunque pueda estar lloviendo más, la vegetación necesita este mes para comenzar a recuperarse. Otro elemento interesante es que en mayo un porcentaje considerable de los incendios son originados por rayos.

De forma general puede decirse que en tanto se llega a la temporada de lluvias van en aumento los valores mensuales medios de ocurrencias de incendios, de las temperaturas y de la velocidad del viento, ocurriendo lo contrario para el caso de la humedad relativa.

La distribución de los valores medios de las variables meteorológicas durante el año y su determinante influencia sobre el combustible vegetal para que esté o no disponible, según se ha tratado anteriormente, es un hecho que determinó la distribución anual de las ocurrencias de incendios y de las áreas quemadas en la provincia Pinar del Río del 2010 al 2014. Resulta importante destacar que la precipitación, además de favorecer el estado de los combustibles vegetales, también incide en su cantidad.

Puede destacarse que en los meses de marzo y abril se registraron los valores promedio más alto de ocurrencias, correspondiendo a abril el mayor valor de las áreas quemadas, el segundo valor más alto de la velocidad del viento y el segundo más bajo de la humedad relativa. En el caso de las precipitaciones corresponde a este mes uno de los valores más bajos. 

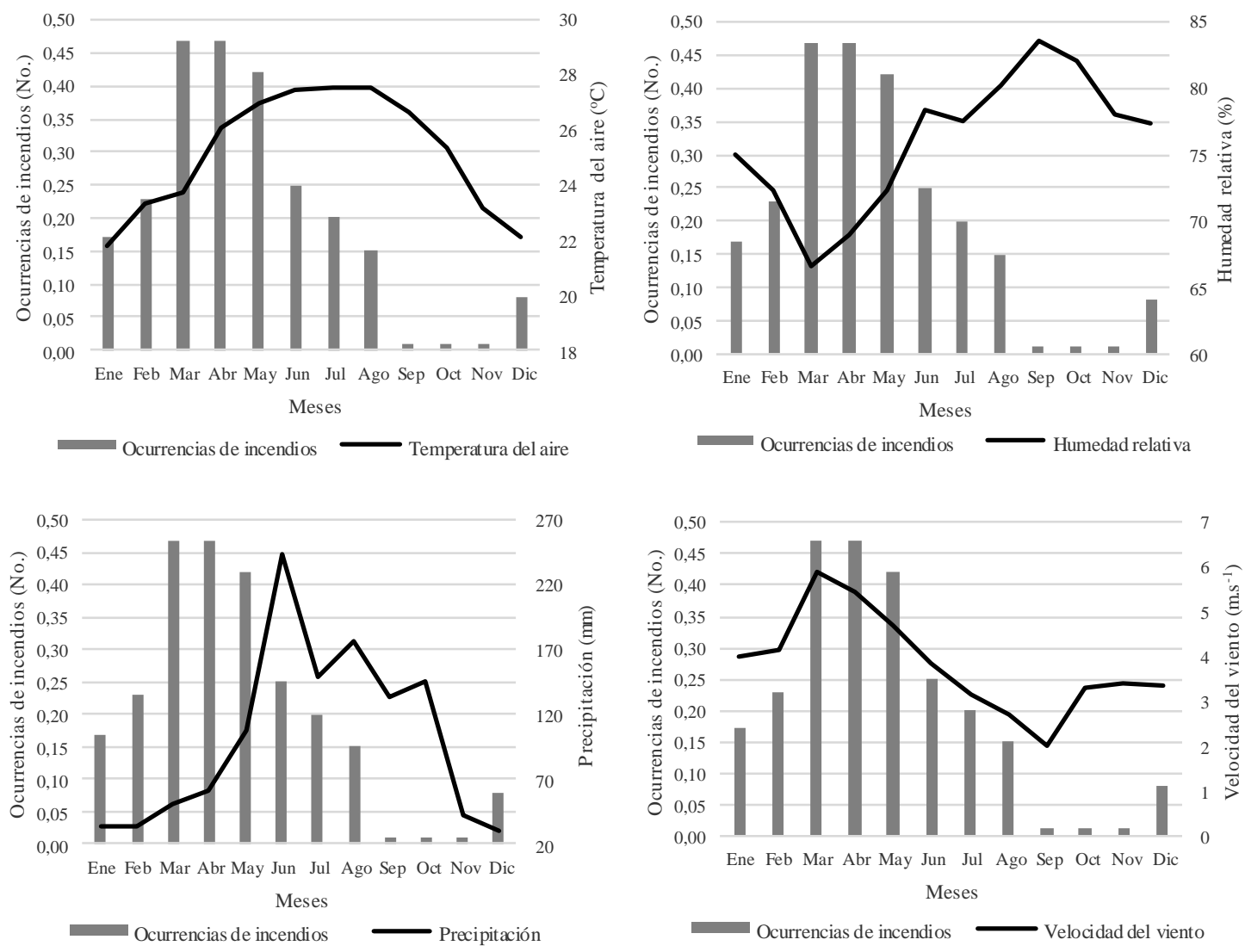

Figura 3. Distribución de las medias mensuales de ocurrencias de incendios, temperatura del aire, humedad relativa, precipitación y velocidad del viento en Pinar del Río (2010-2014).

Figure 3. Distribution of monthly mean of forest fire occurrence, air temperature, relative humidity, rainfall and wind speed in Pinar del Río (2010-2014).

En la tabla 5 se muestran las correlaciones entre variables meteorológicas e incendios forestales. Aunque las correlaciones en todos los casos son significativas ( $\mathrm{p}<0,5)$, las mismas de acuerdo con Bisquerra (2004), pueden clasificarse de bajas y muy bajas. Esto puede ser debido probablemente a la cantidad de datos utilizados y a que se consideraron todos los días del año. Es posible que si se analiza sólo la época de incendios, las correlaciones que involucran variables de incendios habrían sido mayores. Otro elemento a considerar es que se incluyeron tanto a los incendios originados por rayos como los de origen antrópico, causas a las que en la provincia y período objetos de análisis correspondió el 34,30 y 65,70\%, respectivamente, de los mismos.

Tabla 5. Correlación entre variables meteorológicas e incendios forestales.

Table 5. Correlation between meteorological variables and forest fires.

\begin{tabular}{lcc}
\hline Ocurrencias de incendios y variables meteorológicas & $\mathbf{r}$ & $\mathbf{p}$ \\
\hline Ocurrencias de incendios - Temperatura del aire & 0,085 & 0,000 \\
Ocurrencias de incendios - Humedad relativa & $-0,249$ & 0,000 \\
Ocurrencias de incendios - Precipitación & $-0,097$ & 0,000 \\
Ocurrencias de incendios - Velocidad del viento & 0,150 & 0,000 \\
\hline Áreas quemadas y variables meteorológicas & & \\
\hline Áreas quemadas - Temperatura del aire & 0,091 & 0,000 \\
Áreas quemadas - Humedad relativa & $-0,249$ & 0,000 \\
Áreas quemadas - Precipitación & $-0,095$ & 0,000 \\
Áreas quemadas - Velocidad del viento & 0,153 & 0,000 \\
\hline
\end{tabular}

FLORESTA, Curitiba, PR, v. 47, n. 3, p. 343 - 352, jul. / set. 2017

DOI: $10.5380 /$ rf.v47i1.50900 


\section{DISCUSIÓN}

Al comparar el perfil de los incendios forestales de Monte Alegre, Brasil y Pinar del Río, Cuba, Rodríguez et al. (2013) encontraron que la distribución del porcentaje de las ocurrencias de incendios a lo largo del año definió estaciones normales de peligro de incendios diferentes en los dos lugares analizados. Según los mismos autores eso está íntimamente relacionado con la distribución de las variaciones climáticas a lo largo del año en esas regiones, una localizada en el hemisferio sur y otra en el hemisferio norte. En cuanto a la distribución de las ocurrencias de incendios en función del horario de detección, observaron que los incendios ocurrieron principalmente entre as 13:00 y 17:00 horas en las dos regiones. Los autores afirman que ese resultado está relacionado a las variaciones diarias de temperatura y humidad relativa del aire, dos variables del tiempo atmosférico que influencian el material combustible.

La temperatura influye de forma indirecta sobre el comportamiento de los incendios. Según Soares y Batista (2007) el contenido máximo posible de vapor de agua en la atmosfera depende apenas de la temperatura del aire, de ahí que la humedad relativa del aire sea la razón, en porcentaje, entre la cantidad de vapor de agua que el aire contiene y la cantidad máxima que pudiera contener, a la misma temperatura. Los mismos autores plantean que la humedad atmosférica es un elemento clave en la determinación de las condiciones de un potencial incendio. Ella tiene un efecto directo en la inflamabilidad de los combustibles forestales y a través de las interrelaciones con otros factores del tiempo, presenta efectos indirectos sobre algunos aspectos del comportamiento del fuego.

La precipitación es un factor fundamental en la reducción del potencial de ocurrencia de incendios forestales, siendo la estación seca la época en que más ocurren los incendios (SOARES; BATISTA, 2007; WHITE; RIBEIRO, 2011).

El comportamiento obtenido en este trabajo para las precipitaciones, difiere de los resultados reportados por Mbanze et al. (2015) en el Distrito de Lichinga, ubicado al norte de Mozambique, pues mientras que en este estudio los menores valores medios se presentaron de noviembre a abril, en el Distrito de Lichinga esto ocurrió de mayo a octubre. No obstante, los resultados si tienen coincidencia con lo reportado por Rodríguez et al. (2012) en la Empresa Forestal Macurije, ubicada en el territorio de la provincia Pinar del Río, en cuanto a los meses con menores y mayores valores medios.

Al evaluar las ocurrencias de incendios forestales en el Estado de Paraná en el periodo 2005-2010, Tetto et al. (2012) concluyeron que hubo una fuerte correlación entre el número de ocurrencias de incendios y la precipitación.

Viegas y Viegas (1994), analizando la relación entre las precipitaciones y las áreas quemadas en Portugal durante el período 1975-1992, encontraron que las precipitaciones durante el verano, producen un decrecimiento de las áreas quemadas, especialmente si éstas ocurren en el comienzo de la época de incendios.

En este sentido, Govender et al. (2006), en un estudio realizado en África del Sur, obtuvieron que un incremento del $50 \%$ de la media anual de las precipitaciones (desde 500 a $750 \mathrm{~mm}$ ) correspondió con un incremento en la media de la intensidad del fuego del $67 \%$, desde 1500 a $2500 \mathrm{~kW} . \mathrm{m}^{-1}$, reflejando el efecto del incremento de la lluvia sobre la producción de combustible herbáceo. También encontraron que la época de incendios afectó a la intensidad del fuego, principalmente como resultado de los cambios en la humedad del combustible.

Los rayos originaron en la provincia Pinar del Río de 2000 al 2009 el 43,75\% de los incendios (RAMOS-RODRÍGUEZ; CABRERA-REINA, 2011). Resultados similares se obtuvieron para la provincia Pinar del Río, durante diferentes períodos de tiempo, de acuerdo con Rodríguez y Soares (1998). Estos últimos autores encontraron un período de mayores áreas quemadas de marzo a julio. El mayor valor para las áreas quemadas medias por incendios se presentó en abril, mientras que Rodríguez et al. (2008) obtuvieron para la provincia Pinar del Río de 1997 al 2006 el mayor valor para el mes de mayo.

Analizando las correlaciones entre factores meteorológicos e incendios forestales para el municipio de Telêmaco Borba, de 1965 a 1971, Soares (1972) no encontró correlaciones consideradas importantes entre ocurrencias de incendios, áreas quemadas y factores meteorológicos, expresando que la extensión del área quemada no está apenas en función de los factores meteorológicos, pues también depende de los medios de control existentes. No obstante, al hacer los cálculos de las correlaciones sin considerar los incendios por rayos, obtuvo mejor tendencia de las correlaciones entre ocurrencias de incendios y factores meteorológicos.

\section{CONCLUSIONES}

- Los valores medios mensuales registrados en abril fueron estadísticamente diferentes al resto de los meses, con excepción de los valores de las precipitaciones y las ocurrencias de incendios que no tienen diferencia 
con los registrados en marzo. En este contexto se puede considerar que el mes de abril es el más propicio para la ocurrencia de los incendios forestales.

- Durante los meses de mayor cantidad de ocurrencias de incendios y áreas quemadas se registraron los valores medio más altos de la velocidad del viento y los más bajos de la humedad relativa. No obstante, las correlaciones entre variables meteorológicas e incendios forestales, aunque existe, fueron bajas o muy bajas, lo cual sugiere la idea de realizar otras investigaciones similares pero considerando solo la época de incendios y también descartando los incendios originados por rayos.

\section{REFERENCIAS}

BISQUERRA, R. Metodología de la investigación educativa. Madrid: Editorial La Muralla, 2004. 459p.

CARRASCO, Y. Diseño de una fórmula para la determinación del índice de peligro de incendios forestales en la provincia Pinar del Río, Cuba. 141 f. Disertación (Doctorado en Ciencias Forestales) - Universidad de Pinar del Río, Cuba, 2016.

GOVENDER, N.; TROLlOPE, W. S. W.; VAN WILGEN, B. W. The effect of fire season, fire frequency, rainfall and management on fire intensity in savanna vegetation in South Africa. Journal of Applied Ecology, v. 43, p. 748-758, 2006.

LIU, Y.; STANTURF, J.; GOODRICK, S. Trends in global wildfire potential in a changing climate. Forest Ecology and Management, v. 259, p. 685-697, 2010.

MBAnZE, A. A.; BATISTA, A. C.; TETTO, A. F.; KOEHLER, H. S.; MANTEIGA, J. B. Influence of the meteorological conditions on forest fires occurrences in Lichinga District, Northern Mozambique. Revista Floresta, v. 45, n. 3. p. 577-586, 2015.

MOLINA-TERRÉN, D.; CARDIL, A. Temperature determining larger wildland fires in NE Spain. Theorethical and Applied Climatololgy, v. 125, n. 1-2, p. 295-302, 2015.

RAMOS-RODRÍGUEZ, M. P.; CABRERA-REINA, J. M. Los incendios forestales en Pinar del Río, Cuba, del 2000 al 2009. Revista Forestal Baracoa, IIF, Cuba, v. 30, Número Especial, p. 42-53, 2011.

RODRÍGUEZ, M. P. R.; CABRERA, J. M.; ORTEZ, L. A. Eficiencia de la protección contra incendios forestales en Pinar del Río, Cuba. Revista Forestal Baracoa, v. 27, n. 2, p. 31-40, 2008.

RODRÍGUEZ, M. P. R.; SOARES, R. V. Comportamiento histórico de los incendios forestales en la provincia de Pinar del Río, Cuba. Revista Floresta, v. 28, n. 1⁄2, p. 03-18, 1998.

RODRÍGUEZ, M. P. R.; SOARES, R. V.; BATISTA, A. C.; TETTO, A. F.; SIERRA, C. A. M.; RODRÍGUEZ, Y. C. Ajuste e desempenho dos índices de perigo de incêndios Nesterov, FMA e FMA ${ }^{+}$na Empresa Florestal Macurije, Cuba. Revista Floresta, v. 42, n. 4, p. 651-660, 2012.

RODRÍGUEZ, M. P. R.; SOARES, R. V.; BATISTA, A. C.; TETTO, A. F.; MARTíNEZ, L. W. Comparação entre o perfil dos incêndios florestais de Monte Alegre, Brasil, e de Pinar del Río, Cuba. Revista Floresta, Curitiba, v. 43, n. 2, p. 231-240, 2013.

RODRÍGUEZ, M. P. R.; SOARES, R. V.; BATISTA, A. C.; TETTO, A. F.; CABRERA, J. M.; MARTÍNEZ, L. W. Eficiencia de la protección contra incendios forestales en Monte Alegre, Brasil y Pinar del Río, Cuba. Revista Floresta, Curitiba, v. 44, n. 4, p. 617-628, 2014.

SOARES, R. V. Índices de perigo de incêndio. Revista Floresta, Curitiba, v. 3, n. 3, p. 19-40 p., 1972.

SOARES, R. V.; BATISTA, A. C. Incêndios florestais: controle, efeitos e uso do fogo. Curitiba, 2007. 250p.

SOARES, R. V.; BATISTA, A. C.; TETTO, A. F. Meteorologia e climatologia florestal. Curitiba, 2015. 215p.

TETTO, A. F.; BATISTA, A. C.; SOARES, R. V. Ocorrência de incêndios florestais no estado do Paraná, no período de 2005 a 2010. Revista Floresta, Curitiba, v. 42, n. 2, p. 391-398, 2012.

THOMPSON, J. R.; SPIES, T. A. Vegetation and weather explain variation in crown damage within a large mixed-severity wildfire. Forest Ecology and Management, v. 258, p. 1684-1694, 2009.

VIEGAS, D. X.; BOVIO, G.; FERREIRA, A.; NOSENZO, A. Comparative study of various methods of fire danger evaluation in southern Europe. Int. J. Wildland Fire, v. 9, n. 4, p. 235-246, 1999. 
VIEGAS, D. X.; VIEGAS, M. T. A relationship between rainfall and burned area for Portugal. Int. J. Wildland Fire, v. 4, n. 1, p. 11-16, 1994.

WESTERLING, A. L.; BRYANT, B. P. Climate change and wildfire in California. Climate Change, n. 87, Suppl.1, S231-S249, 2008.

WHITE, B. L. A.; RIBEIRO, A. S. Análise da precipitação e sua influência na ocorrência de incêndios florestais no Parque Nacional Serra de Itabaiana, Sergipe, Brasil. Revista Ambiente \& Agua, v. 6, n. 1, p. 148-156, 2011. 